\title{
Stress Cardiomyopathy Due to Status Epilepticus After Fontan Procedure
}

\author{
Preeti Ramachandran ${ }^{1}$, Stephanie Y Tseng ${ }^{2,3}$, Kenneth E Mah ${ }^{2,3}$ and Jonathan W Byrnes ${ }^{2,3,4 *}$ \\ ${ }^{1}$ Division of Pediatric Cardiology, Department of Pediatrics, Kentucky Children's Hospital, University of Kentucky, Kentucky \\ ${ }^{2}$ Department of Pediatrics, University of Cincinnati College of Medicine, Cincinnati, USA \\ ${ }^{3}$ The Heart Institute, Division of Cardiology, Cincinnati Children's Hospital Medical Center, Cincinnati, USA \\ ${ }^{4}$ Department of Pediatrics, University of Alabama Birmingham, Birmingham, USA
}

*Corresponding author: Jonathan W Byrnes, Department of Pediatrics,

University of Cincinnati College of Medicine, Cincinnati, OH, USA.

Key words: Fontan; Cardiomyopathy; Status epilepticus; Resuscitation

\section{Introduction}

This case describes an adolescent with Fontan physiology who had two episodes resulting in severe ventricular dysfunction--with normal ventricular function before and between the two episodes.

Ventricular function recovered rapidly after the first episode with medical management; however, she was not able to recover despite the application of mechanical circulatory support in the second episode. The phenomenon of stress cardiomyopathy has not been described in children with congenital heart disease but given the growing population of with palliated congenital heart disease this diagnosis must be considered in similar cases.

\section{Case Report}

A 13-year-old female with double outlet right ventricle and mitral atresia, post non-fenestrated Fontan palliation and a history of partial complex seizure disorder (controlled with valproic acid and oxcarbazepine) presented to the emergency department (ED) in status epilepticus. She had a typical seizure that became generalized. Emergency medical services were summoned and administered benozodiazepines and bag-mask-ventilated her to the ED.

In the ED, she received fosphenytoin and phenobarbital with resolution of seizures after 90 minutes. Her respiratory status remained tenuous with oxygen saturation less than 80 percent with relative hypotension. A chest X-ray revealed bilateral pulmonary venous congestion. She was urgently intubated for hypoxemia. C-reactive protein, procalcitonin, and white blood cell count were normal.
Valproic acid and oxcarbazepine levels were undetectable.

In the cardiac intensive care unit (CICU), she was tachycardic, had jugular venous distention, and a gallop. Her liver was four centimeters beneath the costal margin and only central pulses were palpable. An echocardiogram showed severely depressed right ventricular systolic function (baseline echocardiogram: normal right ventricular function). A central venous line estimated Fontan pressure at $30 \mathrm{mmHg}$. Inotropes and vasopressors were administered. Video electroencephalogram (EEG) and valproic acid were started.

Seizures did not recur during the hospitalization. Over the next 72 hours, hemodynamic and respiratory support were discontinued, and right ventricular systolic function normalized. A cardiac catheterization on hospital day ten demonstrated elevated Fontan pressure of $24 \mathrm{mmHg}$, elevated trans-pulmonary gradient and low cardiac index. She was discharged 16 days after admission. The working diagnosis at discharge was status epilepticus with associated systolic ventricular dysfunction likely due to stress cardiomyopathy. Her right ventricular function remained normal; however, she presented again 41 months later in status epilepticus.

She had been well until the evening of repeat presentation with status epilepticus, and cyanosis ensued. The family administered intranasal midazolam and initiated cardiopulmonary resuscitation (CPR). Emergency medical services continued the resuscitation. In the ED, seizures were extinguished with levetiracetam and valproic acid. However, she exhibited myoclonus. Due to relative hypotension 
and decreased pulses, fluid resuscitation and epinephrine infusion were applied.

She became pulseless during transport to the CICU, so CPR was resumed. Pulseless electrical activity persisted therefore she was placed on veno-arterial extracorporeal membrane oxygenation (ECMO). Despite maximal ECMO flow and volume resuscitation, she remained hypotensive and non-pulsatile. An echocardiogram demonstrated severely diminished right ventricular function. She remained unresponsive; EEG noted slowing and suppressed background without seizures. She developed fixed and dilated pupils and a head computed tomography demonstrated signs of anoxic injury and uncal herniation. Family withdrew care, and she died 17 hours after seizure onset.

We postulate this patient experienced stress cardiomyopathy generated by the high catecholamine state of status epilepticus; it is confounded by the associated application of anti-epileptic medications in both events. Benzodiazepines have not been implicated unless the doses are supratherapeutic, even when accounting for allometric scaling [1,2]. Thiopental and pentobarbital are two barbituates implicated in direct myocardial depression, but no reports of cardiac toxicity attributable to phenobarbital are available to our knowledge [2,3].

The progression of seizure to respiratory failure, followed by the rapid (< six hours after instituting positive pressure ventilation) resolution of alveolar infiltrates in the first scenario suggests a cardiac contribution to respiratory decompensation. The elevated end diastolic pressure despite normalization of systolic function by echocardiogram suggests subclinical diastolic dysfunction may have placed this patient at risk for the development of pulmonary edema and respiratory compromise once a prolonged high systemic vascular resistance state was encountered. The same physiologic state would have similarly been met during the second presentation.

Stress induced cardiomyopathy is a well-recognized entity in adult literature where certain pathologic conditions such as stroke, seizures, cardiac arrest or sepsis interfere with the normal cerebral-cardiac regulatory mechanisms and result in impaired cardiovascular function [4]. Several variants of stress cardiomyopathy exist based on distribution of ventricular dysfunction. Takotsubo's cardiomyopathy is one such recognized subtype where reversible left ventricular dysfunction occurs in the setting of acute emotional or physical stress or severe illness. Neurogenic stress cardiomyopathy is another reported subtype where ventricular dysfunction is precipitated by acute neurologic complications such as subarachnoid hemorrhage and seizures [5]. Multiple mechanisms have been postulated for stress cardiomyopathies including myocardial stun from coronary microvascular dysfunction, epicardial coronary spasm and catecholamine surge resulting in myocardial injury $[4,6]$. Although these reversible cardiomyopathies typically recover ventricular function within days or weeks, the recognition of these etiologies is important because of hemodynamic instability during the acute phase.
Pediatric case reports describing stress cardiomyopathies are rare [7]. This case is the first known report of status epilepticus triggering stress cardiomyopathy in a single ventricle patient after Fontan palliation resulting in severe ventricular dysfunction. Prompt resolution of decreased systolic function after seizure control and hemodynamic support in the initial presentation makes an argument for stress cardiomyopathy. In the second presentation, inadequate seizure control for an extended period prior to arrival in the CICU may have eliminated the potential for reversibility. We suspect prolonged seizures with respiratory depression may have resulted in a massive catecholamine surge which an "at risk" myocardium could not accommodate because of concurrent hypoxemia and right ventricular geometry. With the ever-expanding population of patients with Fontan circulation and pediatric heart failure, physicians who care for critically ill pediatric patients both in ED and critical care settings must recognize individuals with at risk myocardium. Rapid seizure control and treatment of myocardial dysfunction is paramount.

\section{Conclusion}

This case supports stress cardiomyopathy in a single ventricle patient after Fontan procedure and highlights the importance of involving specialists familiar with congenital cardiac anomalies and cardiopulmonary interactions presentation of early in presentation. Additionally, importance of timely seizure control in patients with Fontan physiology is emphasized.

\section{Acknowledgement}

None.

\section{Conflict of Interest}

The authors do not have any conflicts of interest to disclose.

\section{References}

1. Gelissen HP, Epema AH, Henning RH, Krijnen HJ, Hennis PJ, et al. (1996) Inotropic effects of propofol, thiopental, midazolam, etomidate, and ketamine on isolated human atrial muscle. Anesthesiology 84(2): 397403.

2. Kanaya N, Murray PA, Damron DS (2002) The differential effects of midazolam and diazepam on intracellular $\mathrm{Ca} 2+$ transients and contraction in adult rat ventricular myocytes. Anesth Analg 95(6): 16371644

3. Kolh P, Lambermont B, Ghuysen A, Tchana Sato V, Dogné JM, et al. (2004) Comparison of the effects of propofol and pentobarbital on left ventricular adaptation to an increased afterload. J Cardiovasc Pharmacol 44(3): 294-301.

4. Bybee KA, Prasad A (2008) Stress-related cardiomyopathy syndromes. Circulation 118(4): 397- 409.

5. Lee VH, Oh JK, Mulvagh SL, Wijdicks EF (2006) Mechanisms in neurogenic stress cardiomyopathy after aneurysmal subarachnoid hemorrhage. Neurocrit Care 5(3): 243-249.

6. Zaroff JG, Rordorf GA, Titus JS, Newell JB, Nowak NJ, et al. (2000) Regional myocardial perfusion after experimental subarachnoid hemorrhage. Stroke 31(5): 1136-1143.

7. Finsterer J, Stollberger C (2015) Neurological and non-neurological triggers of Takotsubo syndrome in the pediatric population. Int J Cardiol 179: 345-347. 